\title{
Approximate Circuit Representation of Voltage Unbalance Emission Due to Load Asymmetry in Three-Phase Power Systems
}

\author{
Diego Bellan \\ Department of Electronics, Information and Bioengineering \\ Politecnico di Milano \\ Milan, Italy \\ diego.bellan@polimi.it
}

\begin{abstract}
This work investigates from a circuit viewpoint the phenomenon of voltage unbalance emission due to load asymmetry in three-phase power systems. Voltage unbalance emission occurs whenever a power system shows a lack of structural symmetry between the three phases. In such a case, in fact, the transformation methods, such as the symmetrical component transformation, provide coupled modal circuits. As a result, in a practical three-phase three-wire power system, the positivesequence circuit injects current into the negative-sequence circuit and therefore the load voltage shows a negative-sequence component due to modal circuits interaction. Since in many cases of practical interest the asymmetry level in a three-phase load can be regarded as a small perturbation with respect to the ideal symmetrical condition, in this paper a weak-coupling approach between sequence circuits is proposed. More specifically, load asymmetry is investigated, and analytical conditions for circuit weak-coupling are derived. In this case an approximate equivalent circuit for the estimation of voltage unbalance emission is derived. Analytical results are validated by means of numerical simulation of a radial power system with asymmetrical three-phase load.
\end{abstract}

Keywords-low-frequency electromagnetic compatibility, power quality, power system analysis, power system simulation, transmission lines

\section{INTRODUCTION}

The conventional approach for the analysis of three-phase power systems under steady-state sinusoidal conditions foresees the use of the symmetrical component transformation [1]-[3]. This approach is just a special case of the more general approach consisting in the modal analysis of circuits. Roughly speaking, modal analysis of circuits consists in a proper transformation of voltage/current variables such that the transformed impedance/admittance matrices are diagonalized. Thus, the transformed system can be seen as a collection of uncoupled circuits whose solution can be readily evaluated. The symmetrical component transformation yields three uncoupled circuits (i.e., the positive, negative, and zero sequence circuits) under the fundamental assumption of a three-phase system with perfect structural symmetry between the three phases, i.e., the circuit parameters of the three phases (e.g., resistance, capacitance, self and mutual inductance) must be equal. In practical three-phase power systems, however, this condition can be reached only in an approximate way. The two main reasons leading to three-phase asymmetry are related to the lines and to the loads [4]-[7]. In fact, three-phase lines are usually transposed such that the three conductors can be regarded as geometrically equivalent along the whole length of the line. As far as the loads are concerned, however, the use of large singlephase loads (e.g., high-speed railway lines) can result in a significant asymmetry in a three-phase load. The lack of symmetry in a three-phase load results in a mutual coupling between the sequence circuits after the symmetrical component transformation.

In an ideal three-phase power system the voltage source consists only in the positive sequence component. Therefore, in a symmetrical system, the negative and zero sequence circuits are not excited. If the symmetry assumption is not met, however, coupling between sequence circuits results in injection of current also in the negative and zero sequence circuit. In this process the positive sequence circuit acts as the source of emission, and the negative and zero sequence circuits represent the victims. In many cases, power systems consist only in the interconnection of components with three wires. In such case, the zero sequence circuit is an open circuit (i.e., the common mode is not present). Thus, the victim of the emission is the negative sequence circuit only. Emission of the positive sequence circuit on the negative sequence circuit due to lack of system symmetry is called voltage unbalance emission [8]-[13].

In practical applications it is of paramount importance to limit such phenomenon because it has adverse effects on both supply utilities and customer installations. Three-phase rotating machines are the major victim under unbalanced supply voltages. In particular, the presence of voltage unbalance adversely affects the operation of three-phase induction motors in terms of high unbalanced phase currents, reduced motor torque and speed, increased noise and vibration. Additional heat in windings leads to reduction in the motor efficiency, thus demanding motor de-rating [12]-[13].

It is important to notice that voltage unbalance emission is one of the interference phenomena regarded as low-frequency conducted emission in electromagnetic compatibility (EMC) [14]-[15]. Indeed, EMC investigates electromagnetic phenomena starting from zero hertz. From this viewpoint, 
voltage unbalance emission is one of the main conducted lowfrequency phenomena.

Since in many cases of practical interest the asymmetry level in a three-phase load can be regarded as a small perturbation with respect to the ideal symmetrical condition, in this paper a weak-coupling approach between sequence circuits is proposed. Analytical investigation of the problem provides the conditions to be met such that weak-coupling can be assumed. Thus, in case of weak coupling the emission from the positive to the negative sequence circuit can be readily evaluated through a simple and approximate equivalent circuit consisting in a current-controlled voltage source depending on the load asymmetry.

The paper is organized as follows. In Section II the symmetrical component transformation is recalled. In Section III, the circuit representation of load asymmetry under the assumption of weak coupling between positive and negative sequence circuits is derived. In Section IV the analytical expression of voltage unbalance emission is numerically validated though a Matlab simulation of a radial network. Finally, some concluding remarks are proposed in Section V.

\section{SYMMETRICAL COMPONENT TRANSFORMATION}

The conventional approach for the steady-state analysis of symmetrical three-phase power systems foresees the use of the symmetrical component transformation in the phasor domain. The main advantage of this approach consists in obtaining decoupled equations even if the three phases are coupled in the original variables. This approach is a special case of the more general modal analysis. The three uncoupled modal circuits, called sequence circuits (i.e., positive, negative, and zero sequence circuits), can be readily solved in the sequence domain and the original variables can be recovered by inverse transformation.

The transformation matrix, in its rational form, is defined as

$$
\mathbf{S}=\frac{1}{\sqrt{3}}\left[\begin{array}{ccc}
1 & \alpha & \alpha^{2} \\
1 & \alpha^{2} & \alpha \\
1 & 1 & 1
\end{array}\right]
$$

where

$$
\alpha=e^{j \frac{2}{3} \pi}=-\frac{1}{2}+j \frac{\sqrt{3}}{2}
$$

and $\alpha^{2}=\alpha^{*}$. The transformation matrix is a Hermitian matrix, i.e., $\mathbf{S}^{-1}=\mathbf{S}^{T *}$.

The symmetrical component transformation when applied to phasor voltages provides

$$
\left[\begin{array}{c}
V_{p} \\
V_{n} \\
V_{\mathbf{0}}
\end{array}\right]=\mathbf{S} \cdot\left[\begin{array}{c}
V_{a} \\
V_{b} \\
V_{c}
\end{array}\right]
$$

where $V_{p}, V_{n}$, and $V_{0}$ are the positive, negative, and zero sequence voltages. Of course, the same transformation applies to phasor currents.
Symmetrical three-phase passive components (i.e., lines and loads) can be described in terms of an impedance matrix with the following structure

$$
\mathbf{Z}=\left[\begin{array}{ccc}
Z & Z_{m} & Z_{m} \\
Z_{m} & Z & Z_{m} \\
Z_{m} & Z_{m} & Z
\end{array}\right]
$$

By defining the column vectors

$$
\mathbf{V}_{s}=\left[\begin{array}{c}
V_{p} \\
V_{n} \\
V_{0}
\end{array}\right], \mathbf{I}_{s}=\left[\begin{array}{c}
I_{p} \\
I_{n} \\
I_{0}
\end{array}\right], \mathbf{V}=\left[\begin{array}{c}
V_{a} \\
V_{b} \\
V_{c}
\end{array}\right], \mathbf{I}=\left[\begin{array}{c}
I_{a} \\
I_{b} \\
I_{c}
\end{array}\right]
$$

the transformed current/voltage relationship for a symmetrical passive component can be written

$$
\mathbf{V}_{s}=\mathbf{S Z S}^{-1} \mathbf{I}_{s}=\mathbf{Z}_{s} \mathbf{I}_{s}
$$

where

$$
\mathbf{Z}_{s}=\left[\begin{array}{ccc}
Z_{p} & 0 & 0 \\
0 & Z_{n} & 0 \\
0 & 0 & Z_{0}
\end{array}\right]
$$

and

$$
\begin{gathered}
Z_{p}=Z_{n}=Z-Z_{m} \\
Z_{0}=Z+2 Z_{m}
\end{gathered}
$$

The diagonal form of the sequence impedance matrix (7) leads to the above-mentioned uncoupled sequence circuits when the transformation is applied to the whole three-phase system.

\section{ASYMMETRICAL LOADS}

In many cases of practical interest the equivalent load of a three-phase power system can be modelled as three uncoupled impedances $Z_{a}, Z_{b}$, and $Z_{c}$. The power system is designed such that the three impedances are equal, but in practice a deviation from the nominal impedance level cannot be avoided. By denoting as $Z$ the average value of the load impedances:

$$
Z=\frac{Z_{a}+Z_{b}+Z_{c}}{3}
$$

the three load impedances can be written in terms of the arbitrary deviation (i.e., no symmetrical deviations are required) with respect to such average value:

$$
Z_{a}=Z+\delta Z_{a}, \quad Z_{b}=Z+\delta Z_{b}, \quad Z_{c}=Z+\delta Z_{c}
$$

where, by taking into account (9), the deviations are such that:

$$
\delta Z_{a}+\delta Z_{b}+\delta Z_{c}=0 .
$$

By using the symmetrical component transformation defined in the previous Section, and by considering only the positive and negative sequence circuits (i.e., the common condition of a power system with three wires), the voltage/current relationship (6) can be written as: 


$$
\left[\begin{array}{c}
V_{p} \\
V_{n}
\end{array}\right]=\mathbf{Z}_{\text {sload }}\left[\begin{array}{l}
I_{p} \\
I_{n}
\end{array}\right]=\left[\begin{array}{cc}
Z & \delta Z_{n} \\
\delta Z_{p} & Z
\end{array}\right]\left[\begin{array}{l}
I_{p} \\
I_{n}
\end{array}\right]
$$

where

$$
\begin{aligned}
& \delta Z_{p}=\left(\delta Z_{a}+\alpha \delta Z_{b}+\alpha^{2} \delta Z_{c}\right) / 3 \\
& \delta Z_{n}=\left(\delta Z_{a}+\alpha^{2} \delta Z_{b}+\alpha \delta Z_{c}\right) / 3
\end{aligned}
$$

Notice that $\delta Z_{p}$ and $\delta Z_{n}$ are defined by expressions similar

to $V_{p}$ and $V_{n}$ in (3), respectively (i.e., with $1 / 3$ instead of $1 / \sqrt{3}$ as a multiplicative factor).

Relationships (12)-(13) provide the coupling coefficients between the positive and negative sequence circuits due to an asymmetrical three-phase load. The remaining part of the sequence circuits consist in the same line impedance $Z_{\text {line }}$ since from (8a) the positive and negative sequence line impedances are equal, and the positive/negative components $E_{p}$ and $E_{n}$ of the three-phase source (see Fig. 1).

Voltage unbalance emission of the load consists in the voltage $V_{n}$ in Fig. 1 due to the coupling of the negative sequence circuit with the positive sequence circuit. A rigorous solution of the circuit in Fig. 1 can be obtained by solving a coupled problem. An approximate solution based on a weak coupling assumption, however, would be interesting for engineering applications where simple and approximate results can be usefully exploited to obtain quick solutions of complicated problems. In fact, the procedure outlined in this paper, can be readily extended to the case of a power system with more complicated structure and including several asymmetrical loads.

The problem in Fig. 1 can be seen as an interference problem where the source circuit consists in the positive sequence circuit, and the victim circuit consists in the negative sequence circuit. Assuming weak coupling means that the positive sequence current is not affected by the negative sequence circuit:

$$
I_{p} \cong \frac{E_{p}}{Z_{\text {line }}+Z}
$$

The rigorous solution for $I_{p}$ in Fig. 1, however, is given by:

$$
I_{p}=\frac{E_{p}-\frac{\delta Z_{n}}{Z+Z_{\text {line }}} E_{n}}{Z+Z_{\text {line }}-\frac{\delta Z_{p} \delta Z_{n}}{Z+Z_{\text {line }}}}
$$

By comparing (14) and (15) we obtain the conditions for weak coupling assumption:

$$
\begin{gathered}
\left|\frac{\delta Z_{n}}{Z+Z_{\text {line }}} E_{n}\right| \ll\left|E_{p}\right| \\
\left|\frac{\delta Z_{p} \delta Z_{n}}{\left(Z+Z_{\text {line }}\right)^{2}}\right| \ll 1
\end{gathered}
$$

The constraint (16a) is usually met since in most of practical

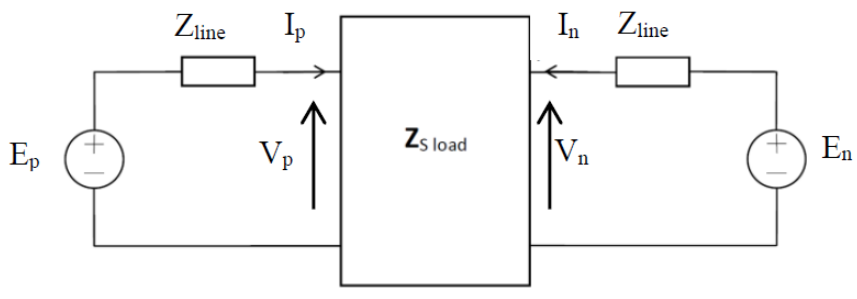

Fig. 1. Coupling of the positive sequence and negative sequence circuits due to an asymmetrical three-phase load.

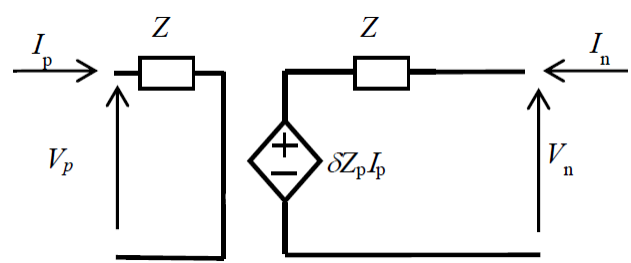

Fig. 2. Approximate circuit representation of the impact of the positive sequence circuit on the negative sequence circuit, based on the weak coupling assumption.

cases $\left|E_{n}\right| \ll\left|E_{p}\right|$. The constraint (16b) should be checked, but it is reasonably expected that it is true in most of the cases where impedance deviations are small. However, when the threephase load shows a large asymmetry (e.g., the case of a large single-phase load) the constraint (16b) could be critical.

It is interesting to notice that even in the case of large impedance deviations, if such deviations show a three-phase symmetry (i.e., $\delta Z_{a}, \delta Z_{b}, \delta Z_{c}$ with equal magnitude and $2 \pi / 3$ relative phase displacement) then $\delta Z_{p}=0$ or $\delta Z_{n}=0$, which means that $(16 b)$ is met.

In case of weak coupling, the positive sequence circuit can be solved by neglecting circuit coupling (i.e., by using (14)). Then the impact on the negative sequence circuit can be represented by a current-controlled voltage source $\delta Z_{p} I_{p}$ (see Fig. 2).

According to Figs. 1 and 2, the approximate expression for the Voltage Unbalance Factor (VUF) based on the weak coupling assumption can be easily derived:

$$
V U F=\left|\frac{V_{n}}{V_{p}}\right|=\left|\frac{\delta Z_{p}}{Z} \cdot \frac{Z_{\text {line }}}{Z+Z_{\text {line }}}\right|
$$

\section{NUMERICAL VALIDATION}

The approximate expression (17) based on the weak coupling assumption (16) can be readily validated by means of numerical simulation (Matlab-Simulink) of a simple radial network consisting in a $12.47 \mathrm{kV}-60 \mathrm{~Hz}$ three-phase ideal source with only positive sequence component, a $3.22 \mathrm{~km}$ symmetrical line, and an asymmetrical load (see Fig. 3) [4]. The line is characterized by the following impedance matrix:

$$
\tilde{\mathbf{Z}}=\left[\begin{array}{lll}
0.2494+j 0.8748 & 0.0592+j 0.4985 & 0.0592+j 0.4985 \\
0.0592+j 0.4985 & 0.2494+j 0.8748 & 0.0592+j 0.4985 \\
0.0592+j 0.4985 & 0.0592+j 0.4985 & 0.2494+j 0.8748
\end{array}\right]\left[\frac{\Omega}{\mathrm{km}}\right]
$$

whereas the average impedance of the load is $Z=10+j 10 \Omega$. 


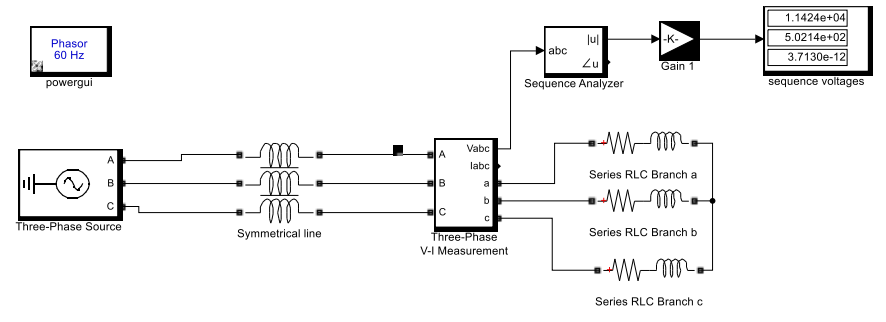

Fig. 3. Simulink implementation of the three-phase power system used to validate to weak coupling model proposed in the paper.

In the first simulation, the impedance deviations $\delta Z_{a}, \delta Z_{b}, \delta Z_{c}$ (with zero mean value) were selected such that $\delta Z_{n}=0$. Therefore, from (16b) the condition for weak coupling holds. The impedance deviations are selected such that $\left|\delta Z_{p}\right|$ ranges from $10 \%$ to $50 \%$ of $Z$. According to Fig. 2, $\delta Z_{p} I_{p}$ is the source of voltage unbalance emission on the negative sequence circuit. Analytical approximate results provided by (17) (solid line) are compared with numerical simulations (crosses) in Fig. 4. Notice that even in the case of $50 \%$ impedance deviation, the weak coupling model works properly since the symmetry of impedance deviations guarantees that condition (16b) is satisfied.

The second numerical example was designed in order to check the weak-coupling condition (16b). Only the resistive component of the three loads was changed such that the average value $10 \Omega$ was kept. To this aim, the resistance of load $a$ was increased from $10 \Omega$ to $30 \Omega$, whereas the resistances of loads $b$ and $c$ were decreased from $10 \Omega$ to zero. In terms of deviation with respect to the average value, the relative deviation of resistance $a$ ranges from zero to 2. In Fig. 5 the weak coupling model (17) (solid line) is compared with Simulink numerical results (crosses). As expected, the accuracy of the weak coupling model decreases as the load asymmetry increases. The ratio in (16b) equals 0.10 for load $a$ deviation equal to 1 (i.e., the midpoint in Fig. 5), whereas the ratio equals 0.42 for load $a$ deviation equal to 2 (i.e., the right-end point in Fig. 5). Notice that even in the last case the approximation provided by the weak coupling model is still reasonable.

\section{CONCLUSION}

An approximate model for the voltage unbalance emission based on the weak coupling assumption for the positive and negative sequence circuits in a three-phase power system with asymmetrical load has been derived and validated. It was shown that under proper hypothesis a load asymmetry can be represented as a current-controlled voltage source in the negative sequence circuit. Validity of the weak coupling assumption allows a straightforward evaluation of the resulting voltage unbalance emission. The proposed result can be interesting in view of a more general application in complex power systems where several asymmetrical loads are present. It is expected that each load asymmetry can be properly represented by the approximate model derived in the paper, and therefore a straightforward evaluation of the combined effects of such asymmetries can be obtained.

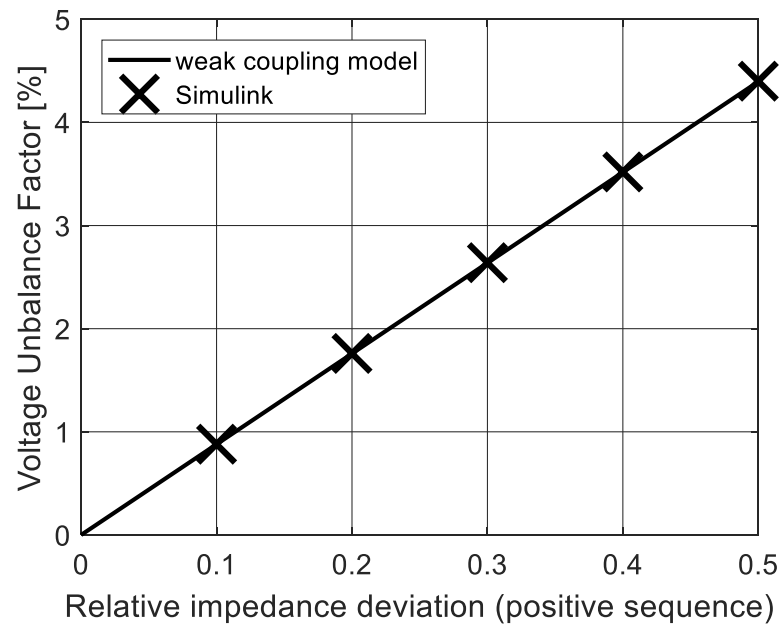

Fig. 4. Comparison between numerical and analytical approximate evaluations of the Voltage Unbalance Factor for the system in Fig. 3. Impedance deviations are symmetrical and such that (13b) is equal to zero (i.e., only the impedance deviation (13a) is different from zero).

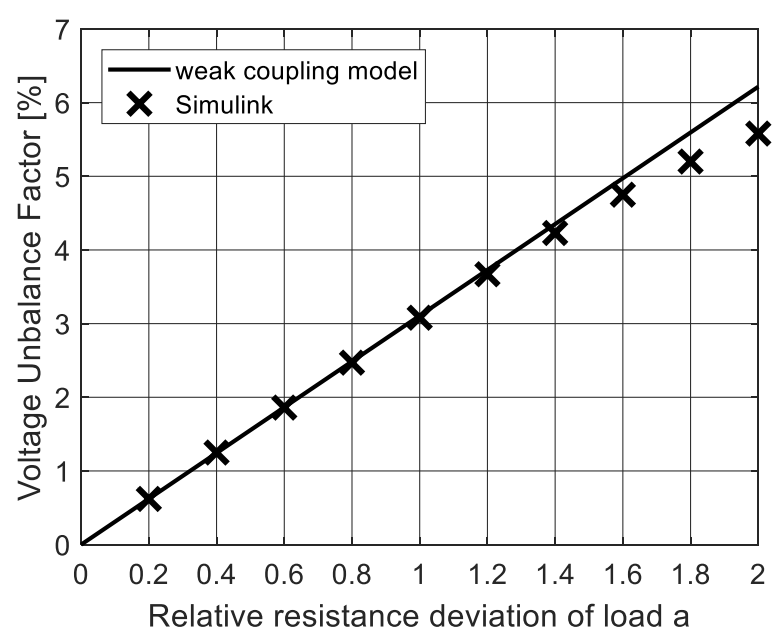

Fig. 5. Comparison between numerical and analytical approximate evaluations of the Voltage Unbalance Factor (for the system in Fig. 3) as a function of the relative deviation of resistance of load $a$ with respect to the average value. The resistances of loads $b$ and $c$ are changed accordingly, in order to hold the average value.

\section{REFERENCES}

[1] C. L. Fortescue, "Method of symmetrical coordinates applied to the solution of polyphase networks," Trans. AIEE, vol. 37, pp. 1027-1140, 1918.

[2] G. Chicco, P. Postolache, and C. Toader, "Analysis of three-phase systems with neutral under distorted and unbalanced conditions in the symmetrical component-based framework," IEEE Trans. on Power Delivery, vol. 22, no.1, pp. 674-683, Jan. 2007.

[3] G. C. Paap, "Symmetrical components in the time domain and their application to power network calculations," IEEE Trans. on Power Systems, vol. 15, no. 2, pp. 522-528, May 2000.

[4] D. Bellan and S. A. Pignari, "A circuit approach for the propagation analysis of voltage unbalance emission in power systems," in Proc. 2016 IEEE PES Asia Pacific Power and Energy Engineering Conference (APPEEC 2016), pp. 1355-1359. 
[5] D. Bellan, G. Superti Furga, and S. A. Pignari, "Circuit representation of load and line asymmetries in three-phase power systems," International Journal of Circuits, Systems and Signal Processing, vol. 9, pp. 75-80, 2015.

[6] D. Bellan and S. A. Pignari, "Circuit representation of voltage unbalance emission due to line asymmetry," in Proc. 2015 IEEE Innovative Smart Grid Technologies - Asia (ISGT ASIA), pp. 1-5.

[7] R. Muzzammel and U. Tahir, "Analytical behaviour of line asymmetries in three phase power systems," in Proc. of IEEE International Symposium on Recent Advances in Electrical Engineering, 24-26 October, 2017, Islamabad, Pakistan.

[8] Z. Emin and D. S. Crisford, "Negative phase-sequence voltages on E\&W transmission system," IEEE Trans. on Power Delivery, vol. 21, no. 3, pp. 1607-1612, July 2006.

[9] P. Paranavithana, S. Perera, R. Koch, and Z. Emin, "Global voltage unbalance in MV networks due to line asymmetries," IEEE Trans. on Power Delivery, vol. 24, no. 4, pp. 2353-2360, Oct. 2009.

[10] T. E. Seiphetlho and A. P. J. Rens, "On the assessment of voltage unbalance," in Proc. $201014^{\text {th }}$ International Conference on Harmonics and Quality of Power, pp. 1-6.
[11] H. Renner, "Voltage unbalance emission assessment," in Proc. Elect. Power Quality Supply Reliability Conf., June 2010, pp. 43-48.

[12] U. Jayatunga, S. Perera, and P. Ciufo, "Voltage unbalance emission assessment in radial power systems," IEEE Trans. Power Delivery, vol. 27, no. 3, pp. 1653-1661, July 2012.

[13] U. Jayatunga, S. Perera, P. Ciufo, and A. P. Agalgaonkar, "Deterministic methodologies for the quantification of voltage unbalance propagation in radial and interconnected networks," IET Generation, Transmission \& Distribution, vol. 9, no. 11, pp. 1069-1076, 2015.

[14] Electromagnetic compatibility (EMC) - limits - assessment of emission limits for the connection of unbalanced installations to $M V, H V$ and $E H V$ power systems, Technical report IEC/TR 61000-3-13, Ed. I, International Electrotechnical Commission, 2008.

[15] M. Olofsson and U. Grape, "Voltage quality in the context of EMC," in Proc. 2009 International Symposium on Electromagnetic Compatibility, Kyoto, Japan, pp. 241-244. 